\title{
Antibiotics as deep modulators of gut microbiota: between good and evil
}

\author{
Gianluca laniro, ${ }^{1}$ Herbert Tilg, ${ }^{2}$ Antonio Gasbarrini ${ }^{1}$
}

${ }^{1}$ Internal Medicine, Gastroenterology and Liver Unit, "Agostino Gemelli" University Hospital, Catholic University of Rome, Italy ${ }^{2}$ Department of Internal Medicine I, Gastroenterology, Endocrinology \& Metabolism, Medical University Innsbruck, Innsbruck, Austria

\section{Correspondence to}

Professor Antonio Gasbarrini, Internal Medicine,

Gastroenterology and Liver Unit, "Agostino Gemelli" University Hospital, Largo A. Gemelli, Rome 00168, Italy; antonio.gasbarrini@unicatt.it

Received 21 May 2016 Revised 21 July 2016 Accepted 22 July 2016 Published Online First 16 August 2016
CrossMark

\footnotetext{
To cite: laniro G, Tilg $H_{\text {, }}$ Gasbarrini A. Gut 2016;65:1906-1915.
}

\begin{abstract}
The recent increase in our knowledge of human gut microbiota has changed our view on antibiotics. Antibiotics are, indeed, no longer considered only beneficial, but also potentially harmful drugs, as their abuse appears to play a role in the pathogenesis of several disorders associated with microbiota impairment (eg, Clostridium difficile infection or metabolic disorders). Both drug-related factors (such as antibiotic class, timing of exposure or route of administration) and host-related factors appear to influence the alterations of human gut microbiota produced by antibiotics. Nevertheless, antibiotics are nowadays considered a reliable therapy for some non-communicable disorders, including IBS or hepatic encephalopathy. Moreover, some antibiotics can also act positively on gut microbiota, providing a so-called 'eubiotic' effect, by increasing abundance of beneficial bacteria. Therefore, antibiotics appear to change, for better or worse, the nature of several disorders, including $I B S, I B D$, metabolic disorders or liver disease. This reviews aims to address the potential of antibiotics in the development of major non-communicable disorders associated with the alteration of gut microbiota and on newly discovered therapeutic avenues of antibiotics beyond the cure of infectious diseases.
\end{abstract}

\section{INTRODUCTION: THE ANTIBIOTIC REVOLUTION AND THE MICROBIOTA REVOLUTION}

The discovery of penicillin by Alexander Fleming in 1928 represents a breakthrough in the history of medicine as it catalysed the development of antibiotics, beginning the so-called 'antibiotic revolution'. ${ }^{1}$ By conferring to mankind resilience to the majority of bacterial assaults, antibiotics altered the natural history of most infectious diseases and saved millions of lives. Their exploitation transformed not only medicine, but also pharmacology, healthcare industry and the whole human everyday life.

Several decades after, another paradigm shift changed again our relationship with bugs. Although Metchnikoff already hypothesised a role for our microbiota in health and disease at the beginning of the 20th century, ${ }^{2}$ the undetectability of most components of gut microbiota with available diagnostic technique impeded the growth of interest in this field. The development of 'omics' technologies, based on microbial genome sequencing, allowed the in-depth assessment of gut microbiota composition and functions, ${ }^{3}$ laying the groundwork to a new approach to microbial components of our ody, recently addressed as 'microbiota revolution'. ${ }^{4}$

After this groundbreaking improvement of technology, two cornerstones of antibiotic therapy

\section{Key messages}

- Antibiotics are no longer considered only beneficial, but also potentially harmful agents, as their overuse has been linked to microbiota impairment and related disorders.

- Antibiotics appear to be a reliable treatment not only for infectious disorders, but also for several non-communicable ones.

- Antibiotic characteristics that can influence their 'evil' or 'good' behaviour towards gut microbiota include their class, pharmacokinetics, pharmacodynamics and range of action, as well as their dosage, duration and administration route.

- Host-related factors that are known to influence the size and quality of antibiotic damage to balanced microbiota include age, lifestyle and microbiota composition.

- Some antibiotics can also act positively on gut microbiota, providing a so-called 'eubiotic' effect, by increasing abundance of beneficial bacteria.

started to be undermined. First, antibiotics are no longer considered only beneficial, but also potentially harmful agents, as increasing evidence supports the correlation between their overuse and the development of many disorders associated with the alteration of gut microbiota; ${ }^{5}$ moreover, antibiotics overexposure may also lead to the development of genotypic antibiotic resistance in the resident microbiota and to its potential transfer to pathogenic bacteria; ${ }^{6}$ additionally, antibiotics were indeed shown to enrich phage-encoded genes, which transfer resistance to both the administered drug and also to unrelated antibiotics, as well as to promote the interplay between phages and bacteria, enhancing, consequently, resistance gene exchange.

The second undermined certainty about antibiotics is that the healing of bacterial infections is not considered anymore as their only therapeutic purpose as they appear to be a reliable treatment for several non-communicable disorders (eg, hepatic encephalopathy (HE) or IBS).

This reviews aims to address the potential of antibiotics in the manipulation of gut microbiota and related clinical consequences, focusing both on GI and extraintestinal disorders associated with microbiota impairment driven by antibiotics, and on newly discovered therapeutic avenues of antibiotics beyond the cure of infectious diseases. 


\section{Key messages}

- Antibiotics appear to change, for better or worse, the natural history of several disorders, including IBD, IBS, metabolic disorders or liver disease.

- Use of antibiotics has been suggested to play a direct role in the development of IBD by leading to dysbiosis and reduced bacterial diversity. Another theory, instead, suggests antibiotics are only surrogate markers of other risk factors for IBD such as GI infections.

- Although systemic antibiotics appear to increase the risk of IBS development, a large body of evidence identified poorly absorbable antibiotics (mainly rifaximin) as a reliable treatment for IBS and hepatic encephalopathy.

- According to the administration of high or low dosages, respectively, antibiotics were shown to be able to drive either to underweight, through deep demolition of gut microbiota, or overweight, by development of selective dysbiosis.

- Early life appears to be a critical period for the maturation of metabolic functions, and antibiotic-dependent impairment of microbiota during this period, even if transient, could have profound effects on weight gain, although recent studies provided conflicting results.

- A wiser use of antibiotics in clinical practice, as well as a boost in this field of research, is advocated for a better management of patients with disorders related to the impairment of gut microbiota. the main, but not the only one, effect of antibiotics on our intestine: other mechanisms of action include a direct harmful effect of antibiotics on GI epithelia and the spread of antibiotic-resistant microorganisms. ${ }^{14}$

Short-term alterations of healthy gut microbiota due to antibiotics have been extensively investigated in the past years. ${ }^{15}$ On the contrary, only a few recent studies have addressed longlasting changes of gut microbiota after antibiotic treatment. Both drug-related factors and host-related factors affect the impact of antibiotics on human gut microbiota.

\section{Drug-related factors}

Antibiotic characteristics that can influence this relationship include their class, pharmacokinetics, pharmacodynamics and range of action, as well as their dosage, duration and administration route. ${ }^{16}$ Different antibiotic classes provide different patterns of microbiota alteration because of their different spectrum and bacterial target (table 1). Lincosamides, mainly clindamycin, are broad-spectrum antibiotics with main biliary excretion (and, consequently, high concentrations in stools), which are particularly active against anaerobes. This feature makes clindamycin one of the strongest antibiotic risk factors for the development of Clostridium difficile infection, with an OR of 2.86, as accounted

Table 1 Overview on the effects of different antibiotics on gut microbiota, according to their classes and excretion

\begin{tabular}{|c|c|c|}
\hline Antibiotic class & Antibiotic excretion & Effects on gut microbiota \\
\hline $\begin{array}{l}\text { Lincosamides } \\
\text { Clindamycin }\end{array}$ & Main biliary excretion & $\begin{array}{l}\downarrow \text { Gram-positive aerobes and } \\
\text { anaerobes }^{17} \\
\uparrow \text { Resistance genes }{ }^{18} \\
\downarrow \text { Bacteroides diversity }\end{array}$ \\
\hline $\begin{array}{l}\text { Macrolides } \\
\text { Clarithromycin } \\
\text { Erythromycin }\end{array}$ & Biliary excretion & $\begin{array}{l}\downarrow \text { Total bacterial diversity } \\
\downarrow \text { Actinobacteria (including } \\
\text { Bifidobacteria) }^{1920} \\
\downarrow \text { Firmicutes (mainly } \\
\text { Lactobacilli) }^{20} \\
\uparrow \text { Bacteroidetes }^{20} \\
\uparrow \text { Proteobacteria }^{20} \\
\downarrow \text { Firmicutes }^{26} \\
\downarrow \text { Actinobacteria }^{26} \\
\uparrow \text { Proteobacteria }^{26}\end{array}$ \\
\hline $\begin{array}{l}\beta \text {-Lactams } \\
\text { Penicillin V } \\
\text { Amoxicillin } \\
\text { Ampicillin/ } \\
\text { sulbactam } \\
\text { Cephalosporins }\end{array}$ & $\begin{array}{l}\text { Main urinary excretion } \\
\text { Partial (33-67\%) biliary } \\
\text { excretion }^{24}\end{array}$ & 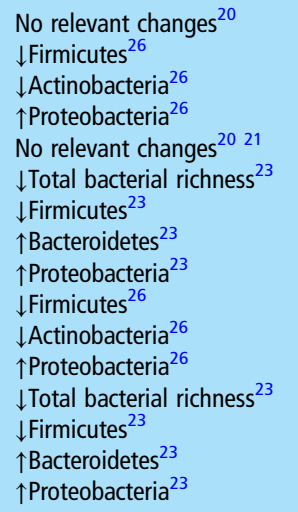 \\
\hline $\begin{array}{l}\text { Fluoroquinolones } \\
\text { Ciprofloxacin } \\
\text { Levofloxacin }\end{array}$ & Partial biliary excretion & $\begin{array}{l}\downarrow \text { Bacterial diversity }{ }^{27} \\
\downarrow \text { Gram-negative facultative } \\
\text { anaerobes }^{25} \\
\uparrow \text { Gram-positive aerobes }^{26} \\
\downarrow \text { Gram-negative facultative } \\
\text { anaerobes } \\
\downarrow \text { Gram-positive anaerobes }\end{array}$ \\
\hline $\begin{array}{l}\text { Glycopeptides } \\
\text { Vancomycin* }\end{array}$ & & $\begin{array}{l}\downarrow \text { Total bacterial diversity } \\
\text { \Firmicutes } \\
{ }^{20} \\
\uparrow \text { Proteobacteria }^{20}\end{array}$ \\
\hline
\end{tabular}

\section{EFFECTS OF ANTIBIOTICS ON GUT MICROBIOTA COMPOSITION}

Human gut microbiota in health

Bacteria are the most represented members of the human gut microbiota, with $>1000$ species, most of which are anaerobes, accounting for an absolute number higher than $10^{14} .8$ Although increasing evidence on gut virome ${ }^{9}$ and gut mycome ${ }^{10}$ is appearing, most of the available data on our microbiota still concern bacteria. The majority $(>90 \%)$ of bacteria identified in human stools and/or intestinal mucosa belong to the Bacteroidetes and Firmicutes phyla. The remaining bacteria are included within four major phyla (Actinobacteria, Fusobacteria, Proteobacteria and Verrucomicrobia) and other minor phyla. ${ }^{11}$ Bacteria are the most relevant contributors in the fulfilment of microbiota-dependent functions, including the regulation of several metabolic pathways, the barrier action against pathogens, and the growth and consolidation of immune system. ${ }^{12}$

As $60-80 \%$ of intestinal bacteria are not identifiable by culture, only the transition to culture-independent technologies, including high-throughput sequencing and other DNA sequencing techniques, lets us discover composition and functions of healthy gut bacterial microbiota. ${ }^{13}$

Consequently, this makeover allowed us to identify the 'microbiota fingerprint' of several disorders, understand the potential role of gut microbiota in their development and learn how microbiota deteriorates under particular conditions, including dietary habits, diseases or drugs. ${ }^{8}$

\section{The effect of antibiotics on gut microbiota composition}

As most of the antibiotics available on the market have a broad spectrum of action, they impact not only on harmful bacteria, but also on healthy ones. The impairment of gut microbiota is 
in a recent meta-analysis. ${ }^{17}$ Clindamycin decreased Gram-positive aerobes and anaerobes, when given for 10 days to healthy individuals, with long-lasting impairment of microbiota composition. ${ }^{18}$ Long-term disturbances of microbiota composition, mainly decrease in the clonal diversity of Bacteroides, and persistent increase in levels of specific resistance genes, were also observed after short clindamycin exposure. ${ }^{19}$

As well as lincosamides, macrolides are protein synthesis inhibitors with biliary excretion, which act mainly against Gram-positive bacteria. Indeed, clarithromycin was accounted for the long-lasting decrease of Actinobacteria (which are naturally resistant to metronidazole) observed in Helicobacter pyloripositive patients exposed to standard first-line triple therapy. ${ }^{20}$

More recently, in a large cohort of Finnish children, macrolides were shown to induce long-term alterations of microbiota, particularly reduction of Actinobacteria (mainly Bifidobacteria), Firmicutes (mainly Lactobacilli) and total bacterial diversity, as well as an increase of relative abundance of Bacteroidetes and Proteobacteria. ${ }^{21}$ In the same cohort, interestingly, penicillinsin particular, penicillin $\mathrm{V}$ and amoxicillin-were not associated with relevant changes of microbiota. More recent data confirmed the weak impact of amoxicillin on human gut microbiota. $^{22}$ The powerlessness of amoxicillin in modulating gut microbiota may explain its ineffectiveness in providing nutritional recovery from severe malnutrition, which was recently observed in a large cohort of African children. ${ }^{23}$ However, a $\beta$-lactam combination, including ampicillin and cephalosporins, led to a decrease in Firmicutes and an increase in Bacteroidetes and Proteobacteria (particularly Enterobacteriaceae, which are commonly resistant to $\beta$-lactam antibiotics) and reduced microbial richness. ${ }^{24}$ This difference in results may be explained by the higher concentration in faeces of cephalosporins than amoxicillin. ${ }^{25}$ Interesting data come from the perinatal administration of $\beta$-lactam antibiotics, with or without other antibiotic classes. Intrapartum antibiotic prophylaxis with penicillin, ampicillin or ampicillin plus erythromycin led to decreased levels of short-chain fatty acids (SCFAs), Firmicutes and Actinobacteria, and to increase in Proteobacteria. ${ }^{26}$

Also, some fluoroquinolones, including ciprofloxacin and moxifloxacin, are partially excreted through the biliary system, and about one-third of their oral intake can be found in faeces. Ciprofloxacin acts against Gram-negative facultative anaerobes, whereas levofloxacin also decreases the number of Gram-positive anaerobes, including Bifidobacteria. ${ }^{27}$ Recently, ciprofloxacin was shown to provide long-term changes-mainly, increasing Gram-positive aerobes-to the microbiota of healthy individuals, ${ }^{28}$ as well as to reduce bacterial diversity. ${ }^{29}$ In another series, fluoroquinolones increased Bacteroidetes and Proteobacteria, without modifying Firmicutes. ${ }^{24}$

The ability of antibiotics to manipulate microbiota can also depend on their route of administration. Vancomycin has shown to be a powerful modulator of microbiota when administered orally as it is not absorbed from the gut. First data come from mouse models, in which vancomycin, together with bacitracin, decreased Bacteroidetes, Firmicutes and total microbiota richness. ${ }^{29}$

In a small randomised-controlled trial of obese males with metabolic syndrome, oral vancomycin reduced bacterial diversity and Firmicutes, increasing Proteobacteria. ${ }^{22}$ Moreover, oral route of administration of antibiotics was recently demonstrated to stimulate the development of antibiotic resistance among healthy microbiota members much more than the intravenous one. ${ }^{30}$

Nevertheless, antibiotics can also act positively on gut microbiota, providing a so-called 'eubiotic' effect. ${ }^{31}$ Some antibiotics were, indeed, demonstrated to stimulate the growth of beneficial bacteria. Nitrofurantoin, a broad-spectrum antibiotic with activity for both Gram-negative and Gram-positive bacteria, provided a temporary increase in Actinobacteria, and mainly in Bifidobacteria, when administered to 61 patients for uncomplicated urinary tract infections. ${ }^{32}$ In another small sample of outpatients with urinary tract infections, nitrofurantoin treatment increased also Fecalibacterium genus. ${ }^{33}$

Increasing evidence supports the 'eubiotic' properties of rifaximin, a poorly absorbable antibiotic with broad-spectrum coverage (aerobes, anaerobes, Gram-positive and Gram-negative bacteria). In a small trial of 15 patients with IBS, rifaximin increased bacterial diversity, the Firmicutes/Bacteroidetes ratio and the abundance of Faecalibacterium prausnitzii, a butyrate producer with strong anti-inflammatory properties, which is reduced in IBD. ${ }^{34}$ In another report of 19 patients with different GI and liver disorders (IBD, IBS, diverticular disease, HE), rifaximin increased the abundance of Lactobacilli, with no effects on bacterial diversity. ${ }^{35}$

Finally, early life treatment with vancomycin was able to prevent the onset of diabetes in non-obese diabetic mice by increasing levels of Akkermansia muciniphila, ${ }^{36}$ a mucindegrading bacterium that can be found in the intestinal mucus layer, whose abundance is inversely correlated with body weight and type 2 diabetes (T2D) both in mice and humans. ${ }^{37} 38$ Interestingly, levels of A muciniphila increased also in two critically ill patients after treatment with multiple antibiotics. ${ }^{39}$

\section{Host-related factors}

Also, host-related factors are known to influence the size and quality of antibiotic damage to healthy bacteria, including age, lifestyle and microbiota composition.

Concerning data come from paediatric studies as infant microbiota is still unconsolidated, so reacts worse to injuries. Antibiotics appear to modify human gut microbiota starting yet from the very early life. Data from several studies support that broad-spectrum antibiotics produce long-lasting effects on microbiota of newborns and infants, including increase in Proteobacteria, and particularly Enterobacteriaceae, and decrease in abundance and/or diversity of Actinobacteria, especially Bifidobacteriaceae and Bifidobacteria. ${ }^{40-42}$ Higher abundance of Enterobacteria was also found in a large cohort of infants treated with antibiotics compared with untreated ones. ${ }^{43}$ Similar results come from data on preterm infants. ${ }^{44}$

A body of evidence shows that antibiotics can impair infant microbiota even before birth. In a large Canadian cohort, infants whose mothers received intrapartum antibiotic prophylaxis displayed a long-lasting dysbiosis, in particular an increase in Enterococci and Clostridia and lower proportion of Bacteroides than others; ${ }^{45}$ under-representation of Bacteroides was found also in infants from a European cohort, whose mothers received antibiotics perinatally and/or during breast feeding. ${ }^{43}$

In summary, available data agree on quite homogeneous alterations of infant microbiota after antibiotic treatment; this is easily explainable as infant microbiota is still immature.

By contrast, the initial composition of gut microbiota appears to influence its response to antibiotics in adults; in a recent study of 18 young healthy volunteers, a 7-day regimen of cefprozil led to similar qualitative alterations of microbiota in the majority of subjects, but to totally different findings in a subset of individuals with predominant Bacteroides enterotype and lower microbial diversity. ${ }^{46}$

Finally, some evidence shows that microbiota response to antibiotics may be also influenced by host lifestyle; in a large cohort of elders, the microbiota of subjects living in long-term care 
residences was more destabilised by antibiotic therapies than that of community-based ones. ${ }^{47}$

Recently, antibiotic effects on gut microbiota have been evaluated through a multi-omic approach. Microbial 16S rDNA and 16S rRNA, metagenome, metatranscriptome, metametabolome and metaproteome of a patient being exposed to cefazolin for 14 days were evaluated; changes in microbiota composition were accompanied by the reduction of the expression of several proteins essential for the microbial activity, by the attenuation of the microbiota metabolic status and by the decrease of hostmicrobial interaction. ${ }^{48}$

\section{A clinical model of antibiotic-driven dysbiosis: the case of C difficile infection}

$C$ difficile infection (CDI) represents probably the most striking clinical example of antibiotic-driven dysbiosis as it usually arises as a complication of antibiotic therapy, especially in elder people. In recent years, the prevalence of CDI has increased tremendously, especially in Western countries, ${ }^{49}$ making it become the leading cause of infectious diarrhoea among hospitalised patients, and, consequently, an economic and healthcare burden. ${ }^{50}$

Overexposure to antibiotics, especially in elderly and fragile patients, has strongly contributed to the development of such a scenario. In a recent meta-analysis pooling >56 000 patients, antibiotics were the highest risk factor for the development of CDI (OR 6.18; 95\% CI 3.80 to 10.04$). .^{51}$

Antibiotic exposure can promote the development of CDI through several pathogenic pathways. First, antibiotic-dependent alteration of healthy microbiota prevents its resistance towards colonisation by C difficile. ${ }^{52}$ Subjects who developed CDI after antibiotic therapy showed a depletion of their microbiota diversity, together with qualitative microbial alterations, including a decrease in families producing SCFAs, such as Lachnospiraceae and Ruminococcaceae. ${ }^{54}$ Also, changes in the metabolic functions of gut microbiota may promote the development of CDI; in a mouse model, the antibiotic-dependent release of free sialic acid by commensal microbiota promoted the colonisation. ${ }^{56}$ Moreover, as production of $C$ difficile toxin is regulated through quorum signalling, ${ }^{57}$ antibiotic-dependent depletion of other microbiota members provides opportunity for $C$ difficile to spread and express its virulence factors.

Finally, growth of $C$ difficile could be promoted also by an 'evil' interplay between microbiota, antibiotics and bile acids. In health, some primary bile acids do not undergo ileal reuptake and can be found in the large bowel; here, cholic acid is able to promote growth of $\mathrm{C}$ difficile ${ }^{58}$ while chenodeoxycholic acid, and the secondary bile acids (lithocholic and deoxycholic acid) derived from microbial-dependent dehydroxylation of primary bile acids, counteract this effect by inhibiting the germination of $C$ difficile spores. ${ }^{59} 60$ Therefore, antibiotics may enhance the growth of $C$ difficile by depleting bacteria that dehydroxylate bile acids, such as Clostridium scindens, which was shown to have a protective role against CDI. ${ }^{60} 61$

The therapeutic corollary of the pathogenic role of antibiotics in CDI is represented by faecal microbiota transplantation, which was proven to be a highly effective treatment for recurrent $\mathrm{CDI},{ }^{62}{ }^{63}$ and to restore the CDI-dependent microbiota impairment. ${ }^{63} 64$

\section{EFFECTS OF ANTIBIOTICS ON NON-COMMUNICABLE DISORDERS}

IBD

Gut microbiota of patients with IBD goes through several qualitative and quantitative alterations. Decrease in bacterial diversity and higher bacterial instability have been described in patients with IBD compared with healthy subjects. ${ }^{65}$ Most pronounced changes in the gut bacteriome of patients with IBD include, at phylum level, increase in Proteobacteria ${ }^{66-68}$ and decrease in Firmicutes. ${ }^{66}$ Among Proteobacteria, increase in Enterobacteriaceae family, ${ }^{66}$ and, in particular, in Escherichia coli adherent-invasive strains, ${ }^{69}$ is the most notable.

Changes in Firmicutes include, beyond reduction of Lactobacilli, $^{70}$ depletion of SCFAs producers, as Lachnospiraceae $^{66}$ and Ruminococcaceae ${ }^{71}$ at family level, and Eubacterium, ${ }^{70}$ Roseburia ${ }^{72}$ and Faecalibacterium, ${ }^{72}$ at genus level; in particular, the decrease of $F$ prausnitzii, an antiinflammatory and butyrate producer strict anaerobe, has been repeatedly found both in patients with Crohn's disease $(\mathrm{CD})^{7475}$ and in patients with UC. ${ }^{75}$

Interesting data come also from the analysis of non-bacterial members of microbiota, previously neglected. Altered fungal diversity, increase of Basidiomycota/Ascomycota ratio and Candida albicans abundance, together with reduction of Saccharomyces cerevisiae abundance, have been reported in patients with IBD. ${ }^{76}$ Also, the 'gut virome' is known to be altered in subjects with IBD: increased richness of bacteriophages, with marked expansion of Caudovirales and specific viral signature for patients with UC and CD, has been recently described; interestingly, it was inversely correlated with decreased bacterial diversity. ${ }^{77}$

Based on these findings, antibiotics have been suggested to play a direct role in the development of IBD by leading to dysbiosis and reduced bacterial diversity. ${ }^{78} \mathrm{~A}$ considerable body of evidence supports this hypothesis. A recent meta-analysis of 11 observational studies and 7208 patients showed that antibiotic exposure has a 1.57 OR for IBD overall (95\% CI 1.27 to 1.94 ); with a significant association for CD (OR 1.74, 95\% CI 1.35 to 2.23 ), especially in children (OR $2.75,95 \%$ CI 1.72 to 4.38 ), but not for UC (OR 1.08, 95\% CI 0.91 to 1.27); all antibiotics, particularly metronidazole (OR 5.01, 95\% CI 1.65 to 15.25 ) and fluoroquinolones (OR 1.79, 95\% CI 1.03 to 3.12), were associated with IBD development, except to penicillin; ${ }^{79}$ these results are in agreement with the weak dysbiotic behaviour of penicillins. $^{21-23}$

Another theory, instead, suggests antibiotics to be only surrogate markers of other risk factors for IBD as bacterial intestinal infections have been identified as a risk factor for further initiation of IBD, especially CD. ${ }^{80}$

In contrast, there are several therapeutic pathways through which antibiotics can change positively the natural course of IBD, including the reduction of luminal bacteria abundance and translocation, and the 'eubiotic' modulation of microbiota. ${ }^{81}$ Many meta-analyses of randomised-controlled trials showed a significant benefit of antibiotics over placebo in the induction of remission of $\mathrm{UC}^{82-84}$ and $\mathrm{CD},{ }^{83}$ although they pooled together different antibiotic classes, making their findings difficult to be interpreted.

Antibiotics are, indeed, suggested by current european crohn's and colitis organisation (ECCO) guidelines for UC only if infectious complications are suspected or ongoing and before surgical interventions. ${ }^{85}$ ECCO-European Society for Paediatric Gastroenterology Hepatology and Nutrition (ESPGHAN) guidelines for the management of paediatric IBD confirmed these advices, without considering antibiotics as a reasonable treatment for the management of uncomplicated paediatric UC. ${ }^{86}$ Also for CD, current ECCO guidelines do not recommend the use of antibiotics for uncomplicated diseases, but only in the case of sepsis, abdominal or perianal abscess, or bacterial overgrowth. ${ }^{87}$ Also, British Society of Gastroenterology (BSG) 
guidelines suggest the use of antibiotics mainly for secondary complications in IBD; nevertheless, the use of antibiotics in short-term treatment of colonic CD is taken into account for patients with refractory disease or contraindications to other therapeutic options supported by stronger evidence. ${ }^{88}$

\section{IBS}

Several lines of evidence support a pathogenic role for impaired microbiota in IBS. First, a considerable rate of patients (up to 30\%) experience IBS symptoms after an episode of acute gastroenteritis (the so-called 'postinfectious IBS'), ${ }^{89}$ especially if happened during early life, ${ }^{90}$ when microbiota is still consolidating. Then, small intestinal bacterial overgrowth has been suggested to play a role in the development of IBS symptoms, ${ }^{91}$ although the issue is still controversial. $^{92}$ Finally, the quite satisfactory success of therapeutic modulators of microbiota, including dietary advices, antibiotics and probiotics, represents an ex juvantibus support of this hypothesis. $^{93}$

Many studies, carried on through DNA sequencing technologies, showed that microbiota composition is altered in subjects with IBS. An increase of Firmicutes/Bacteroidetes ratio was described in patients with IBS compared with controls; in particular, a decrease in the abundance of Bifidobacteria, Faecalibacteria and methanogenic bacteria was found. ${ }^{94}$ Moreover, pyrosequencing analysis identified two microbiotabased subcategories of patients with IBS, including one without abnormalities and another which displayed a decrease in bacterial taxa belonging to Bacteroidetes and an increase in those belonging to Firmicutes. ${ }^{95}$ Interestingly, a depletion of butyrate producers, including Roseburia-Eubacterium rectale group, and an increase in sulfate-reducing bacteria were identified in subjects with constipation-type IBS (C-IBS). ${ }^{96}$

In another analysis of five groups, including patients with postinfectious IBS, patients with or without IBS symptoms 6 months after gastroenteritis, patients with diarrhoea-type IBS (IBS-D) and healthy controls, authors identified a microbiota pattern of 27 genus-like groups of bacteria, which grouped significantly patient groups and controls; in particular, patients displayed a 12-fold increase of Bacteroidetes, and healthy individuals showed a 35 -fold increase in uncultured Clostridia; interestingly, microbiota of patients with postinfectious IBS was similar to that of patients with IBS-D, suggesting a shared pathophysiological pathway. ${ }^{97}$

IPatients with BS exhibit also deep changes in their metabolite profiles, as observed in children with IBS, who showed an increase in proteolysis, incomplete anaerobic fermentation and alterations in the production of methane. ${ }^{98}$ A meta-analysis of case-control studies determining microbiota profiles in patients with IBS showed that IBS-associated species and specific microbiota alterations were influenced by geographical location, being different between Chinese and non-Chinese subjects. ${ }^{99}$

The alteration of gut microbiota has been postulated to contribute to IBS pathogenesis through several pathways, including the impairment of gut-brain axis, of the intestinal barrier and of neuro-enteric physiology. ${ }^{100}$

Antibiotics are able to interfere with the natural history of IBS with a two-faced behaviour, as showed by various lines of evidence. Data from large cohort and case-control studies suggest antibiotics to be a risk factor for the development of IBS and other functional GI disorders (FGIDs), probably by leading to dysbiosis in the host.

In a large retrospective study of nearly 26000 patients, exposure to macrolides or tetracyclines were significant predictors of developing IBS, whereas treatment with cephalosporins or penicillins did not. ${ }^{101}$

In a prospective case-control study, antibiotic therapy for extraintestinal infections was significantly associated with FGIDs (OR 1.90; 95\% CI 1.21 to 2.98) and IBS alone (OR 2.30; CI 1.22 to 4.33$).^{102}$

Although systemic antibiotics appear to increase the risk of IBS development, a large body of evidence identified poorly absorbable antibiotics as a reliable treatment for IBS. At first, neomycin was proven to be effective in improving overall symptoms of IBS together with the normalisation of lactulose breath test, as shown in a large randomised placebo-controlled trial. ${ }^{103}$ Nevertheless, neomycin lost its early attractiveness because of the rapid development of antimicrobial resistance. Absorbable antibiotics, including doxycycline or ampicillin/clavulanate, did not exert reliable results. ${ }^{104}$ Among non-adsorbable antibiotics, rifaximin was the most studied in patients with IBS, and also the one that gave most reliable results, as observed in several randomised, placebo-controlled trials. Rifaximin was significantly more effective than placebo in improving global IBS symptoms when given at the dosage of $400 \mathrm{mg}$ twice daily for 10 days. ${ }^{105}$ Furthermore, rifaximin confirmed to provide sustained improvement of overall IBS symptoms, which lasted for up to 10 weeks after the discontinuation of treatment, at a slightly higher dosage (400 mg thrice daily for 10 days). ${ }^{106}$

However, the strongest evidence about the effectiveness of rifaximin in relieving IBS symptoms comes from two identical randomised double-blind, placebo-controlled trials (TARGET 1 and TARGET 2, respectively). Overall, 1260 patients with IBS without constipation were randomised to placebo or rifaximin at the dosage of $550 \mathrm{mg}$ thrice daily for 2 weeks; rifaximin was significantly more effective than placebo in providing a significant relief of overall IBS symptoms, abdominal pain, bloating and loose or watery stools during the first four weeks after the end of treatment, and a sustained improvement during the first two months and during all 10 weeks of follow-up, in both trials. ${ }^{107}$ This large body of evidence led rifaximin to be approved by US Food and Drug Administration for the treatment of IBS-D in 2015. ${ }^{108}$ Rifaximin and neomycin were investigated also in patients with C-IBS: in a randomised placebo-controlled trial, patients with C-IBS and methane producing were randomised to undergo neomycin with placebo or rifaximin for 14 days; combination therapy achieved better results than neomycin alone, improving significantly constipation, bloating and straining, together with decrease in breath methane. ${ }^{109}$

\section{Obesity-related disorders}

Gut microbiota is known to play a major role in collecting, storing and spending diet-derived energy. As demonstrated by both animal models ${ }^{110}$ and human studies, ${ }^{111}$ the microbiota 'fingerprint' of obesity is represented by an increase in relative abundance of Firmicutes and decrease in that of Bacteroidetes, and the Bacteroidetes/Firmicutes ratio has been addressed as the 'adiposity index', although with some controversies. ${ }^{112}$ Furthermore, a decreased beta diversity, together with a reduction of anti-inflammatory bacteria (such as $F$ prausnitzii) and an increase in pro-inflammatory bacteria (including Ruminococcus gnavus) were observed in obese patients. ${ }^{113}$ Beyond obesity, gut microbiota is altered in other disorders associated with metabolic imbalance; this depends, at least in part, by the direct influence of high-fat diet on gut microbiota, independently of obesity. $^{114}$ 
Recently, two metagenome-wide association studies, respectively from China ${ }^{115}$ and Europe, ${ }^{116}$ addressed microbiota composition in patients with T2D: despite population-specific changes, several common findings, including a decrease in butyrate producers (including Roseburia intestinalis and $F$ prausnitzii), an increase in Proteobacteria and in microbial genes responsible for oxidative stress, were identified. ${ }^{117}$

Moreover, a growing body of evidence suggests that $A$ muciniphila levels are inversely associated with obesity and T2D both in mice and in humans. ${ }^{37} 38$

Recently, a microbiota-dependent therapeutic pathway of the antidiabetic drug metformin has been demonstrated as it has been shown to increase the production of SCFAs in patients with T2D. ${ }^{118}$ In particular, in high-fat diet-fed mice, the metformin-dependent improvement of glycaemic profile appears to be mediated by the increase in A muciniphila levels. ${ }^{119}$

Manipulation of microbiota through antibiotics has been used to modulate weight, also ahead of its time. Farm animals were usually treated with low dosage of antibiotics, to promote their growth, since the 1940s. ${ }^{120}$ Several studies, performed both in animal models and in humans, show that antibiotic treatment have a profound influence on recipients' weight.

In mouse models, effects of antibiotic treatment towards weight and microbiota appear to depend on several factors, including drug dosage and timing of antibiotic exposure. ${ }^{121}$ According to the administration of high or low dosages, respectively, antibiotics were shown to be able to drive either to underweight, through deep demolition of gut microbiota, ${ }^{122}$ or overweight, by development of selective dysbiosis. ${ }^{123} 124$ Beyond total body weight increase, low-dosage antibiotics provide also an increase in adiposity and insulin resistance, alteration of liver metabolism and composition of microbiota. ${ }^{123} 125$

Nonetheless, both in ob/ob and diet-induced obese mice with insulin resistance, antibiotics showed, respectively, antidiabetic effects (improvement in fasting glycaemia, glucose intolerance and insulin signalling, and increase in glucagon-like peptide 1), ${ }^{29126127}$ and decrease of metabolic endotoxemia. ${ }^{128}$

These effects appear to be independent of antibiotic classes, ${ }^{124}$ but also to be deeply influenced by timing of administration: in the study from Cox et al, newborn mice undergoing antibiotic treatment experienced higher weight gain than those receiving antibiotics at weaning and controls. Furthermore, these long-lasting modifications were provided by short-term antibiotic regimens. Early life, therefore, appears to be a critical period for the maturation of metabolic functions, and antibioticdependent impairment of microbiota during this period, even if transient, can have profound effects on weight gain. ${ }^{124}$

Data provided by these elegant mouse models are in agreement with those coming from their human counterpart. A body of large population-based studies, indeed, addressed that early antibiotic exposure (0-24 months of life) is associated with higher risk of overweight/obesity/weight gain later in childhood. ${ }^{129-132}$ These results have been confirmed also in a recent systematic review, which found any antibiotic exposure during infancy as a risk factor for overweight later in childhood. ${ }^{133}$ Nevertheless, some recent papers did not confirm the association between early-life antibiotic administration and later weight gain. ${ }^{23} 134$ Differences in populations (including age and geography) and in drugs features (antibiotic classes, dosages, number of courses) may explain this disagreement. The importance of early-life exposure in driving the metabolic effects of antibiotics has been confirmed by data on antibiotic administration during pregnancy ${ }^{135}$ and adolescence, ${ }^{136}$ which respectively confirm and disagree with those observed in infant population.
Moreover, antibiotics appear to influence also other metabolic pathways, beyond weight gain. A short-term regimen of vancomycin was shown to reduce peripheral insulin sensitivity and microbiota diversity, together with a decrease in Firmicutes and an increase in Proteobacteria, in obese patients, ${ }^{22}$ although these findings were not confirmed in a further study. ${ }^{137}$ Finally, in a large case-control study, a positive association between multiple antibiotic courses (cephalosporins, penicillin, macrolides and quinolones) and the risk of developing diabetes was observed. $^{138}$

In summary, the weight increase and metabolic impairment due to low-dosage antimicrobial therapy may be the result of a phylogenetic trick of human beings: it can be hypothesised that, during ancient times, foods with intrinsic small antimicrobial properties could have driven humans to weight increase and, consequently, low-grade inflammation due to obesity. This should be considered an advantage in conditions of undernourishment and low availability of resources. Considering also the positive correlation between early-life antibiotic exposure and further development of allergic asthma, ${ }^{139-141}$ the use of antibiotics in newborns should be pondered with care by physicians.

\section{Liver disease}

More than two-thirds of the blood directed to the liver comes from the gut, through the portal venous system; this makes quite obvious that a close relationship between gut and liver, the so-called 'gut-liver' axis, exists. In particular, the blood flow drives to the liver several microbial derivates, including peptidoglycans and endotoxins. Gut microbiota plays, indeed, a major role in the pathogenesis of many chronic liver diseases, as supported by increasing evidence. Gut microbiota is known to produce endogenous alcohol and to contribute to non-alcoholic fatty liver disease (NAFLD). ${ }^{142}$ According to different studies, several changes in gut microbiota composition have been described in patients with NAFLD, sometimes with conflicting findings, depending on methods and features of patients and liver disease; ${ }^{143}{ }^{144}$ interestingly, microbiota fingerprints of NAFLD severity have been recently identified, as increase in Bacteroides and Ruminococcus was respectively associated with non-alcoholic steatohepatitis (NASH) and with high levels of liver fibrosis. ${ }^{145}$ Finally, altered intestinal permeability has been described in patients with NAFLD. ${ }^{146}$ Inadequate alcohol intake is also known to alter gut microbiota composition, and increase in Proteobacteria and depletion of Bacteroidetes, and their correlation with endotoxaemia, was observed in alcohol-dependent patients. ${ }^{147} 148$ Finally, two studies of patients with liver cirrhosis showed an increase in pro-inflammatory bacterial families, especially Enterobacteriaceae and Streptococcaceae, and a decrease in beneficial bacteria, including Bifidobacteria and Lachnospiraceae; in particular, Child-Turcotte-Pugh score correlated positively with Streptococcaceae abundance and negatively with Lachnospiraceae abundance. ${ }^{149}$ More recently, quantitative metagenomics revealed decrease in Bacteroidetes and Firmicutes phyla, and increase in Streptococcus and Veillonella genera, in those patients. ${ }^{150}$ Gut microbiota is also known to influence the development of $\mathrm{HE}$ by producing ammonia and eliciting an endotoxin-dependent inflammatory response, which act in synergy. ${ }^{151}$ Cirrhotic patients with HE display higher abundance of Enterobacteriaceae and Alcaligenaceae than both subjects with cirrhosis without $\mathrm{HE}$ and healthy controls; moreover, levels of Porphyromonadaceae and Alcaligenaceae, which produce ammonia by urea degradation, are associated with poor cognitive performance. $^{152}$ 
As a large body of evidence proved their efficacy, antibiotics are considered a cornerstone in the management of HE. After early attempts with neomycin ${ }^{153}$ and metronidazole, ${ }^{154}$ the most relevant data come from randomised-controlled trials on rifaximin.

Rifaximin, given with or without lactulose, showed higher efficacy than lactulose alone in the treatment of patients with overt $\mathrm{HE}^{155}$ and was also more effective than placebo in maintaining remission from HE, ${ }^{156}{ }^{157}$ showing also good safety data. $^{157}$

Moreover, rifaximin achieved reliable efficacy results in the treatment of minimal $\mathrm{HE}^{158}$ and also in the prophylaxis of overt $\mathrm{HE}$ in patients with acute variceal bleeding. ${ }^{159}$

These clinical data have been recently supported by a strong microbiological background: rifaximin has been demonstrated to influence microbiota functions in patients with minimal HE. In particular, despite non-significant changes in microbiota composition (higher abundance of Eubacteriaceae and reduction of Veillonellaceae), treatment with rifaximin provided a significant increase in serum saturated and unsaturated fatty acids, together with reduction in microbiome-metabolome network connections, in particular those involving Enterobacteriaceae, Porphyromonadaceae and Bacteroidaceae, without impairing those regarding autochthonous taxa. These results were also associated with improved cognitive performance in these patients. ${ }^{160}$ This striking evidence let the FDA approve rifaximin against the recurrence of overt $\mathrm{HE}$ or in patients intolerant of lactulose. ${ }^{161}$

\section{CONCLUSIONS}

For better and worse, antibiotics have been shown to be able to affect significantly gut microbiota composition, and consequently, to lead to consequent clinical manifestations, either with a 'eubiotic' effect or a 'dysbiotic' effect. Therefore, the modulation of gut microbiota should be considered a new, striking therapeutic avenue to be used not only for infectious diseases, but also for all other disorders associated with the impairment of gut microbiota. Anyway, antibiotics should be also considered as a double-edged sword since their overuse can drive to harmful clinical consequences. A wiser use of antibiotics in clinical practice, as well as a boost in this field of research, is advocated for a better management of patients with disorders related to the impairment of gut microbiota.

Twitter Follow Gianluca laniro at @gianluca1aniro

Contributors Drafting of the manuscript; critical revision of the manuscript for important intellectual content: all authors.

Funding $\mathrm{HT}$ is supported by the excellence initiative (Competence Centers for Excellent Technologies-COMET) of the Austrian Research Promotion Agency FFG: Research Center of Excellence in Vascular Ageing Tyrol, VASCage (K-Project Nr. 843536) funded by the BMVIT, BMWFW, the Wirtschaftsagentur Wien and the Standortagentur Tirol.

Competing interests None declared.

Provenance and peer review Commissioned; externally peer reviewed.

\section{REFERENCES}

1 Brown K. Penicillin man: Alexander Fleming and the antibiotic revolution. Stroud: Sutton Pub., 2004

2 Metchnikoff E, Mitchell PCS. The prolongation of life: optimistic studies. London: William Heinemann, 1907.

3 Lepage $P$, Leclerc $M C$, Joossens $M$, et al. A metagenomic insight into our gut's microbiome. Gut 2013;62:146-58.

4 Blaser MJ. The microbiome revolution. J Clin Invest 2014;124:4162-5.

5 Blaser MJ. Antibiotic use and its consequences for the normal microbiome. Science 2016;352:544-5
6 Sullivan A, Edlund C, Nord CE. Effect of antimicrobial agents on the ecological balance of human microflora. Lancet Infect Dis 2001;1:101-14.

7 Karami N, Hannoun C, Adlerberth I, et al. Colonization dynamics of ampicillin-resistant Escherichia coli in the infantile colonic microbiota. J Antimicrob Chemother 2008;62:703-8.

8 Sommer F, Bäckhed F. The gut microbiota-masters of host development and physiology. Nat Rev Microbiol 2013;11:227-38.

9 Scarpellini $E$, laniro G, Attili $F$, et al. The human gut microbiota and virome: Potential therapeutic implications. Dig Liver Dis 2015;47:1007-12.

10 laniro G, Bruno G, Lopetuso $L$, et al. Role of yeasts in healthy and impaired gut microbiota: the gut mycome. Curr Pharm Des 2014;20:4565-9.

11 Rajilić-Stojanović M, de Vos WM. The first 1000 cultured species of the human gastrointestinal microbiota. FEMS Microbiol Rev 2014;38:996-1047.

12 Arumugam $\mathrm{M}$, Raes J, Pelletier $\mathrm{E}$, et al. Enterotypes of the human gut microbiome. Nature 2011;473:174-80.

13 Scanlan PD, Marchesi JR. Micro-eukaryotic diversity of the human distal gut microbiota: qualitative assessment using culture-dependent and -independent analysis of faeces. ISME J 2008;2:1183-93.

14 Morgun A, Dzutsev A, Dong $X$, et al. Uncovering effects of antibiotics on the host and microbiota using transkingdom gene networks. Gut 2015;64: 1732-43.

15 Modi SR, Lee HH, Spina CS, et al. Antibiotic treatment expands the resistance reservoir and ecological network of the phage metagenome. Nature 2013:499:219-22.

16 Jernberg C, Löfmark S, Edlund C, et al. Long-term impacts of antibiotic exposure on the human intestinal microbiota. Microbiology (Reading, Engl) 2010;156:3216-23.

17 Slimings C, Riley TV. Antibiotics and hospital-acquired Clostridium difficile infection: update of systematic review and meta-analysis. J Antimicrob Chemother 2014;69:881-91.

18 Rashid MU, Zaura E, Buijs MJ, et al. Determining the long-term effect of antibiotic administration on the human normal intestinal microbiota using culture and pyrosequencing methods. Clin Infect Dis 2015;60(Suppl 2):S77-84.

19 Jernberg C, Löfmark S, Edlund C, et al. Long-term ecological impacts of antibiotic administration on the human intestinal microbiota. ISME J 2007;1:56-66.

20 Jakobsson $\mathrm{HE}$, Jernberg $\mathrm{C}$, Andersson AF, et al. Short-term antibiotic treatment has differing long-term impacts on the human throat and gut microbiome. PLOS ONE 2010;5:e9836.

21 Korpela K, Salonen A, Virta LJ, et al. Intestinal microbiome is related to lifetime antibiotic use in Finnish pre-school children. Nat Commun 2016;7: 10410.

22 Vrieze $A$, Out $C$, Fuentes $S$, et al. Impact of oral vancomycin on gut microbiota, bile acid metabolism, and insulin sensitivity. J Hepatol 2014;60:824-31.

23 Isanaka S, Langendorf C, Berth $\uparrow F$, et al. Routine Amoxicillin for Uncomplicated Severe Acute Malnutrition in Children. N Engl J Med 2016;374:444-53.

24 Knecht $\mathrm{H}$, Neulinger SC, Heinsen FA, et al. Effects of $\beta$-lactam antibiotics and fluoroquinolones on human gut microbiota in relation to Clostridium difficile associated diarrhea. PLOS ONE 2014:9:e89417.

25 Patel IH, Kaplan SA. Pharmacokinetic profile of ceftriaxone in man. Am J Med 1984:77:17-25.

26 Arboleya S, Sánchez B, Solís G, et al. Impact of prematurity and perinatal antibiotics on the developing intestinal microbiota: a functional inference study. Int J Mol Sci 2016;17:pii: E649.

27 Inagaki $Y$, Nakaya R, Chida T, et al. The effect of levofloxacin, an optically-active isomer of ofloxacin, on fecal microflora in human volunteers. Jpn J Antibiot 1992;45:241-52.

28 Dethlefsen L, Relman DA. Incomplete recovery and individualized responses of the human distal gut microbiota to repeated antibiotic perturbation. Proc Natl Acad Sci USA 2011;108(Suppl 1):4554-61.

29 Hwang I, Park YJ, Kim YR, et al. Alteration of gut microbiota by vancomycin and bacitracin improves insulin resistance via glucagon-like peptide 1 in diet-induced obesity. FASEB J 2015;29:2397-411.

30 Zhang L, Huang $Y$, Zhou $Y$, et al. Antibiotic administration routes significantly influence the levels of antibiotic resistance in gut microbiota. Antimicrob Agents Chemother 2013;57:3659-66.

31 Galdston I. Eubiotic medicine. Science 1944:100:76.

32 Vervoort J, Xavier BB, Stewardson A, et al. Metagenomic analysis of the impact of nitrofurantoin treatment on the human faecal microbiota. J Antimicrob Chemother 2015;70:1989-92.

33 Stewardson AJ, Gaïa N, François P, et al. Collateral damage from oral ciprofloxacin versus nitrofurantoin in outpatients with urinary tract infections: a culture-free analysis of gut microbiota. Clin Microbiol Infect 2015;21:344.e1-11.

34 Soldi S, Vasileiadis S, Uggeri F, et al. Modulation of the gut microbiota composition by rifaximin in non-constipated irritable bowel syndrome patients: a molecular approach. Clin Exp Gastroenterol 2015:8:309-25.

35 Ponziani FR, Scaldaferri F, Petito V, et al. The role of antibiotics in gut microbiota modulation: the eubiotic effects of rifaximin. Dig Dis 2016;34: 269-78. 
36 Hansen $\mathrm{CH}$, Krych L, Nielsen DS, et al. Early life treatment with vancomycin propagates Akkermansia muciniphila and reduces diabetes incidence in the NOD mouse. Diabetologia 2012;55:2285-94.

37 Everard A, Belzer C, Geurts L, et al. Cross-talk between Akkermansia muciniphila and intestinal epithelium controls diet-induced obesity. Proc Natl Acad Sci USA 2013;110:9066-71.

38 Dao MC, Everard A, Aron-Wisnewsky J, et al. Akkermansia muciniphila and improved metabolic health during a dietary intervention in obesity: relationship with gut microbiome richness and ecology. Gut 2016;65:426-36.

39 Dubourg G, Lagier JC, Armougom F, et al. High-level colonisation of the human gut by Verrucomicrobia following broad-spectrum antibiotic treatment. Int J Antimicrob Agents 2013;41:149-55.

40 Fouhy $F$, Guinane $C M$, Hussey $S$, et al. High-throughput sequencing reveals the incomplete, short-term recovery of infant gut microbiota following parenteral antibiotic treatment with ampicillin and gentamicin. Antimicrob Agents Chemother 2012;56:5811-20

41 Mangin I, Suau A, Gotteland M, et al. Amoxicillin treatment modifies the composition of Bifidobacterium species in infant intestinal microbiota. Anaerobe 2010;16:433-8.

42 Tanaka S, Kobayashi T, Songjinda P, et al. Influence of antibiotic exposure in the early postnatal period on the development of intestinal microbiota. FEMS Immunol Med Microbiol 2009;56:80-7.

43 Fallani M, Young D, Scott J, et al. Intestinal microbiota of 6-week-old infants across Europe: geographic influence beyond delivery mode, breast-feeding, and antibiotics. J Pediatr Gastroenterol Nutr 2010;51:77-84.

44 Greenwood C, Morrow AL, Lagomarcino AJ, et al. Early empiric antibiotic use in preterm infants is associated with lower bacterial diversity and higher relative abundance of Enterobacter. J Pediatr 2014;165:23-9.

45 Azad MB, Konya T, Persaud RR, et al. Impact of maternal intrapartum antibiotics, method of birth and breastfeeding on gut microbiota during the first year of life: a prospective cohort study. BJOG 2016;123:983-93.

46 Raymond F, Ouameur $A A, D \uparrow$ raspe $M$, et al. The initial state of the human gut microbiome determines its reshaping by antibiotics. ISME J 2016;10:707-20.

47 Jeffery IB, Lynch DB, O'Toole PW. Composition and temporal stability of the gut microbiota in older persons. ISME J 2016;10:170-82.

$48 \mathrm{P} \uparrow$ rez-Cobas AE, Gosalbes MJ, Friedrichs A, et al. Gut microbiota disturbance during antibiotic therapy: a multi-omic approach. Gut 2013;62:1591-601.

49 Lessa FC, Mu Y, Bamberg WM, et al. Burden of Clostridium difficile infection in the United States. N Engl J Med 2015;372:825-34.

50 McGlone SM, Bailey RR, Zimmer SM, et al. The economic burden of Clostridium difficile. Clin Microbiol Infect 2012;18:282-9.

51 Furuya-Kanamori L, Stone JC, Clark J, et al. Comorbidities, exposure to medications, and the risk of community-acquired clostridium difficile infection: a systematic review and meta-analysis. Infect Control Hosp Epidemiol 2015;36:132-41.

52 Reeves $\mathrm{AE}$, Theriot $\mathrm{CM}$, Bergin IL, et al. The interplay between microbiome dynamics and pathogen dynamics in a murine model of Clostridium difficile infection. Gut Microbes 2011:2:145-58.

53 Buffie CG, Jarchum I, Equinda $M$, et al. Profound alterations of intestinal microbiota following a single dose of clindamycin results in sustained susceptibility to Clostridium difficile-induced colitis. Infect Immun 2012;80:62-73.

54 Chang JY, Antonopoulos DA, Kalra A, et al. Decreased diversity of the fecal microbiome in recurrent Clostridium difficile-associated diarrhea. J Infect Dis 2008;197:435-8.

55 Antharam VC, Li EC, Ishmael A, et al. Intestinal dysbiosis and depletion of butyrogenic bacteria in Clostridium difficile infection and nosocomial diarrhea. J Clin Microbiol 2013:51:2884-92.

$56 \mathrm{Ng} \mathrm{KM}$, Ferreyra JA, Higginbottom SK, et al. Microbiota-liberated host sugars facilitate post-antibiotic expansion of enteric pathogens. Nature 2013:502:96-9.

57 Darkoh C, DuPont HL, Norris SJ, et al. Toxin synthesis by Clostridium difficile is regulated through quorum signaling. MBio. 2015;6:e02569.

58 Sorg JA, Sonenshein AL. Bile salts and glycine as cogerminants for Clostridium difficile spores. J Bacteriol 2008;190:2505-12.

59 Sorg JA, Sonenshein AL. Chenodeoxycholate is an inhibitor of Clostridium difficile spore germination. J Bacteriol 2009;191:1115-17

60 Buffie CG, Bucci V, Stein RR, et al. Precision microbiome reconstitution restores bile acid mediated resistance to Clostridium difficile. Nature 2015;517:205-8.

61 Sorg JA, Sonenshein AL. Inhibiting the initiation of Clostridium difficile spore germination using analogs of chenodeoxycholic acid, a bile acid. J Bacteriol 2010;192:4983-90.

62 Cammarota G, Masucci L, laniro G, et al. Randomised clinical trial: faecal microbiota transplantation by colonoscopy vs. vancomycin for the treatment of recurrent Clostridium difficile infection. Aliment Pharmacol Ther 2015;41:835-43.

63 van Nood E, Vrieze A, Nieuwdorp M, et al. Duodenal infusion of donor feces for recurrent Clostridium difficile. N Engl J Med 2013;368:407-15.

64 Weingarden AR, Chen C, Bobr A, et al. Microbiota transplantation restores norma fecal bile acid composition in recurrent Clostridium difficile infection. Am J Physiol Gastrointest Liver Physiol 2014;306:G310-9.
65 Cammarota $G$, laniro $G$, Cianci $R$, et al. The involvement of gut microbiota in inflammatory bowel disease pathogenesis: potential for therapy. Pharmacol Ther 2015;149:191-212.

66 Frank DN, St Amand AL, Feldman RA, et al. Molecular-phylogenetic characterization of microbial community imbalances in human inflammatory bowel diseases. Proc Natl Acad Sci USA 2007;104:13780-5.

67 Walker AW, Sanderson JD, Churcher C, et al. High-throughput clone library analysis of the mucosa-associated microbiota reveals dysbiosis and differences between inflamed and non-inflamed regions of the intestine in inflammatory bowel disease. BMC Microbiol. 2011;11:7.

68 Blachier F, Davila AM, Mimoun S, et al. Luminal sulfide and large intestine mucosa: friend or foe? Amino Acids 2010;39:335-47.

69 Martinez-Medina M, Aldeguer X, Lopez-Siles M, et al. Molecular diversity of Escherichia coli in the human gut: new ecological evidence supporting the role of adherent-invasive E. coli (AIEC) in Crohn's disease. Inflamm Bowel Dis 2009;15:872-82

70 Ott SJ, Musfeldt M, Wenderoth DF, et al. Reduction in diversity of the colonic mucosa associated bacterial microflora in patients with active inflammatory bowel disease. Gut 2004;53:685-93.

71 Lepage $P$, Häsler R, Spehlmann ME, et al. Twin study indicates loss of interaction between microbiota and mucosa of patients with ulcerative colitis. Gastroenterology 2011;141:227-36.

72 Willing BP, Dicksved J, Halfvarson J, et al. A pyrosequencing study in twins shows that gastrointestinal microbial profiles vary with inflammatory bowel disease phenotypes. Gastroenterology 2010;139:1844-54

73 Rajilić-Stojanović M, Shanahan F, Guarner F, et al. Phylogenetic analysis of dysbiosis in ulcerative colitis during remission. Inflamm Bowel Dis 2013;19: 481-8.

74 Sokol H, Pigneur B, Watterlot $L$, et al. Faecalibacterium prausnitzii is an anti-inflammatory commensal bacterium identified by gut microbiota analysis of Crohn disease patients. Proc Natl Acad Sci USA 2008;105:16731-6.

75 Sokol H, Seksik P, Furet JP, et al. Low counts of Faecalibacterium prausnitzii in colitis microbiota. Inflamm Bowel Dis 2009:15:1183-9.

76 Sokol $H$, Leducq V, Aschard $H$, et al. Fungal microbiota dysbiosis in IBD. Gut Published Online First: 3 Feb 2016. doi:10.1136/gutjnl-2015-310746

77 Norman JM, Handley SA, Baldridge MT, et al. Disease-specific alterations in the enteric virome in inflammatory bowel disease. Cell 2015;160:447-60.

78 Gevers D, Kugathasan S, Denson LA, et al. The treatment-naïve microbiome in new-onset Crohn's disease. Cell Host Microbe 2014;15:382-92.

79 Ungaro R, Bernstein CN, Gearry R, et al. Antibiotics associated with increased risk of new-onset Crohn's disease but not ulcerative colitis: a meta-analysis. Am J Gastroenterol 2014;109:1728-38.

80 García Rodríguez LA, Ruigomez A, Pan $\uparrow$ s. Acute gastroenteritis is followed by an increased risk of inflammatory bowel disease. Gastroenterology 2006;130:1588-94.

81 Sartor RB. Therapeutic manipulation of the enteric microflora in inflammatory bowel diseases: antibiotics, probiotics, and prebiotics. Gastroenterology 2004:126:16201633.

82 Rahimi R, Nikfar S, Rezaie A, et al. A meta-analysis of antibiotic therapy for active ulcerative colitis. Dig Dis Sci 2007:52:2920-5.

83 Khan KJ, Ullman TA, Ford AC, et al. Antibiotic therapy in inflammatory bowel disease: a systematic review and meta-analysis. Am J Gastroenterol 2011:106:661-73.

84 Wang SL, Wang ZR, Yang CQ. Meta-analysis of broad-spectrum antibiotic therapy in patients with active inflammatory bowel disease. Exp Ther Med 2012:4:1051-6.

85 Dignass A, Van Assche G, Lindsay JO, et al. The second European evidence-based Consensus on the diagnosis and management of Crohn's disease: Current management. J Crohns Colitis 2010:4:28-62.

86 Turner D, Levine A, Escher JC, et al. Management of pediatric ulcerative colitis: joint ECCO and ESPGHAN evidence-based consensus guidelines. J Pediatr Gastroenterol Nutr 2012;55:340-61.

87 Dignass A, Lindsay JO, Sturm A, et al. Second European evidence-based consensus on the diagnosis and management of ulcerative colitis part 2: current management. J Crohns Colitis 2012;6:991-1030.

88 Mowat C, Cole A, Windsor A, et al, IBD Section of the British Society of Gastroenterology. Guidelines for the management of inflammatory bowel disease in adults. Gut 2011;60:571-607.

89 Thabane M, Kottachchi DT, Marshall JK. Systematic review and meta-analysis: the incidence and prognosis of post-infectious irritable bowel syndrome. Aliment Pharmacol Ther 2007;26:535-44.

90 Cremon C, Stanghellini V, Pallotti F, et al. Salmonella gastroenteritis during childhood is a risk factor for irritable bowel syndrome in adulthood. Gastroenterology 2014;147:69-77.

91 Shah ED, Basseri RJ, Chong K, et al. Abnormal breath testing in IBS: a meta-analysis. Dig Dis Sci 2010;55:2441-9.

92 Simr $\uparrow n$ M, Stotzer PO. Use and abuse of hydrogen breath tests. Gut 2006;55:297-303. 
93 Ianiro G, Bibbò S, Gasbarrini A, et al. Therapeutic modulation of gut microbiota: current clinical applications and future perspectives. Curr Drug Targets 2014;15:762-70.

94 Rajilić-Stojanović M, Biagi E, Heilig HG, et al. Global and deep molecular analysis of microbiota signatures in fecal samples from patients with irritable bowel syndrome. Gastroenterology 2011;141:1792-801.

95 Jeffery IB, O'Toole PW, Öhman L, et al. An irritable bowel syndrome subtype defined by species-specific alterations in faecal microbiota. Gut 2012;61:997-1006.

96 Chassard C, Dapoigny M, Scott KP, et al. Functional dysbiosis within the gut microbiota of patients with constipated-irritable bowel syndrome. Aliment Pharmacol Ther 2012;35:828-38.

97 Jalanka-Tuovinen J, Salojärvi J, Salonen A, et al. Faecal microbiota composition and host-microbe cross-talk following gastroenteritis and in postinfectious irritable bowel syndrome. Gut 2014;63:1737-45.

98 Shankar V, Homer D, Rigsbee L, et al. The networks of human gut microbe-metabolite associations are different between health and irritable bowel syndrome. ISME J 2015;9:1899-903.

$99 \operatorname{Simr} \uparrow n$ M, Barbara G, Flint HJ, et al. Intestinal microbiota in functional bowe disorders: a Rome foundation report. Gut 2013;62:159-76.

100 Zhuang $X$, Xiong L, Li L, et al. Alterations of gut microbiota in patients with irritable bowel syndrome: A systematic review and meta-analysis. J Gastroenterol Hepato/ Published Online First: 14 Jun 2016. doi:10.1111/jgh.13471

101 Villarreal AA, Aberger FJ, Benrud R, et al. Use of broad-spectrum antibiotics and the development of irritable bowel syndrome. WMJ 2012;111:17-20.

102 Paula H, Grover M, Halder SL, et al. Non-enteric infections, antibiotic use, and risk of development of functional gastrointestinal disorders. Neurogastroenterol Motil 2015:27:1580-6.

103 Pimentel M, Chow EJ, Lin HC. Normalization of lactulose breath testing correlates with symptom improvement in irritable bowel syndrome. A double-blind, randomised, placebo-controlled study. Am J Gastroenterol 2003;98:412-19.

104 Yang J, Lee HR, Low K, et al. Rifaximin versus other antibiotics in the primary treatment and retreatment of bacterial overgrowth in IBS. Dig Dis SCi 2008;53:169-74.

105 Sharara Al, Aoun E, Abdul-Baki $\mathrm{H}$, et al. A randomised double-blind placebo-controlled trial of rifaximin in patients with abdominal bloating and flatulence. Am J Gastroenterol 2006;101:326-33.

106 Pimentel M, Park S, Mirocha J, et al. The effect of a nonabsorbed oral antibiotic (rifaximin) on the symptoms of the irritable bowel syndrome: a randomised trial. Ann Intern Med 2006;145:557-63.

107 Pimentel M, Lembo A, Chey WD, et al. Rifaximin therapy for patients with irritable bowel syndrome without constipation. N Engl J Med 2011;364:22-32.

108 http://www.accessdata.fda.gov/scripts/cder/ob/docs/obdetail.cfm?Appl_ $\mathrm{No}=022554 \& \mathrm{TABLE} 1=0 \mathrm{~B}$ RX

109 Pimentel M, Chang C, Chua KS, et al. Antibiotic treatment of constipation-predominant irritable bowel syndrome. Dig Dis Sci 2014;59:1278-85.

110 Ley RE, Bäckhed F, Turnbaugh P, et al. Obesity alters gut microbial ecology. Proc Natl Acad Sci USA 2005;102:11070-5.

111 Ley RE, Turnbaugh PJ, Klein S, et al. Microbial ecology: human gut microbes associated with obesity. Nature 2006;444:1022-3.

112 Duncan SH, Lobley GE, Holtrop G, et al. Human colonic microbiota associated with diet, obesity and weight loss. Int J Obes (Lond) 2008;32:1720-4.

113 Le Chatelier E, Nielsen T, Qin J, et al. Richness of human gut microbiome correlates with metabolic markers. Nature 2013;500:541-6.

114 Hildebrandt MA, Hoffmann C, Sherrill-Mix SA, et al. High-fat diet determines the composition of the murine gut microbiome independently of obesity. Gastroenterology 2009;137:1716-24.e1-2.

115 Qin J, Li Y, Cai Z, et al. A metagenome-wide association study of gut microbiota in type 2 diabetes. Nature 2012;490:55-60.

116 Karlsson FH, Tremaroli V, Nookaew I, et al. Gut metagenome in European women with normal, impaired and diabetic glucose control. Nature 2013;498:99-103.

117 Tilg H, Moschen AR. Microbiota and diabetes: an evolving relationship. Gut 2014:63:1513-21.

118 Forslund K, Hildebrand F, Nielsen T, et al. Disentangling type 2 diabetes and metformin treatment signatures in the human gut microbiota. Nature 2015;528:262-6.

119 Shin NR, Lee JC, Lee HY, et al. An increase in the Akkermansia spp. population induced by metformin treatment improves glucose homeostasis in diet-induced obese mice. Gut 2014;63:727-35.

120 Cromwell GL. Why and how antibiotics are used in swine production. Anim Biotechnol 2002;13:7-27.

121 Cox LM, Blaser MJ. Antibiotics in early life and obesity. Nat Rev Endocrinol 2015;11:182-90.

122 Murphy EF, Cotter PD, Hogan A, et al. Divergent metabolic outcomes arising from targeted manipulation of the gut microbiota in diet-induced obesity. Gut 2013;62:220-6.

123 Cho I, Yamanishi S, Cox L, et al. Antibiotics in early life alter the murine colonic microbiome and adiposity. Nature 2012;488:621-6.
124 Cox LM, Yamanishi S, Sohn J, et al. Altering the intestinal microbiota during a critical developmental window has lasting metabolic consequences. Cell 2014;158:705-21.

125 Mahana D, Trent CM, Kurtz ZD, et al. Antibiotic perturbation of the murine gut microbiome enhances the adiposity, insulin resistance, and liver disease associated with high-fat diet. Genome Med 2016;8:48.

126 Membrez $M$, Blancher $F$, Jaquet $M$, et al. Gut microbiota modulation with norfloxacin and ampicillin enhances glucose tolerance in mice. FASEB J 2008;22:2416-26.

127 Carvalho BM, Guadagnini D, Tsukumo DM, et al. Modulation of gut microbiota by antibiotics improves insulin signalling in high-fat fed mice. Diabetologia 2012:55:2823-34.

128 Cani PD, Bibiloni R, Knauf $C$, et al. Changes in gut microbiota control metabolic endotoxemia-induced inflammation in high-fat diet-induced obesity and diabetes in mice. Diabetes 2008;57:1470-81.

129 Ajslev TA, Andersen CS, Gamborg M, et al. Childhood overweight after establishment of the gut microbiota: the role of delivery mode, pre-pregnancy weight and early administration of antibiotics. Int J Obes (Lond) 2011;35:522-9.

130 Bailey LC, Forrest CB, Zhang P, et al. Association of antibiotics in infancy with early childhood obesity. JAMA Pediatr 2014;168:1063-9.

131 Scott Fl, Horton DB, Mamtani R, et al. Administration of antibiotics to children before age 2 years increases risk for childhood obesity. Gastroenterology 2016;151:120-129.e5.

132 Mbakwa CA, Scheres L, Penders J, et al. Early life antibiotic exposure and weight development in children. J Pediatr Published Online First: 8 Jul 2016. doi:10.1016/ j.jpeds.2016.06.015

133 Woo Baidal JA, Locks LM, Cheng ER, et al. Risk factors for childhood obesity in the first 1,000 days: a systematic review. Am J Prev Med 2016;50:761-79.

134 Gerber JS, Bryan M, Ross RK, et al. Antibiotic exposure during the first 6 months of life and weight gain during childhood. JAMA 2016;315:1258-65.

135 Mueller NT, Whyatt R, Hoepner L, et al. Prenatal exposure to antibiotics, cesarean section and risk of childhood obesity. Int J Obes (Lond) 2015;39:665-70.

136 Contopoulos-loannidis DG, Ley C, Wang W, et al. Effect of long-term antibiotic use on weight in adolescents with acne. $J$ Antimicrob Chemother 2016;71:1098-105.

137 Reijnders D, Goossens GH, Hermes GD, et al. Effects of gut microbiota manipulation by antibiotics on host metabolism in obese humans: a randomized double-blind placebo-controlled trial. Cell Metab 2016;24:63-74.

138 Boursi B, Mamtani R, Haynes K, et al. The effect of past antibiotic exposure on diabetes risk. Eur J Endocrinol 2015;172:639-48.

139 Örtqvist AK, Lundholm C, Kieler $\mathrm{H}$, et al. Antibiotics in fetal and early life and subsequent childhood asthma: nationwide population based study with sibling analysis. BMJ 2014;349:g6979.

140 Sun W, Svendsen ER, Karmaus WJ, et al. Early-life antibiotic use is associated with wheezing among children with high atopic risk: a prospective European study. J Asthma 2015;52:647-52.

141 Lee E, Kwon JW, Kim HB, et al. Association Between Antibiotic Exposure, Bronchiolitis, and TLR4 (rs1927911) Polymorphisms in Childhood Asthma. Allergy Asthma Immunol Res 2015;7:167-74.

142 Zhu L, Baker RD, Zhu R, et al. Gut microbiota produce alcohol and contribute to NAFLD. Gut 2016;65:1232.

143 Mouzaki M, Comelli EM, Arendt BM, et al. Intestinal microbiota in patients with nonalcoholic fatty liver disease. Hepatology 2013;58:120-7.

144 Michail S, Lin M, Frey MR, et al. Altered gut microbial energy and metabolism in children with non-alcoholic fatty liver disease. FEMS Microbiol Ecol 2015;91: $1-9$.

145 Boursier J, Mueller O, Barret M, et al. The severity of nonalcoholic fatty liver disease is associated with gut dysbiosis and shift in the metabolic function of the gut microbiota. Hepatology 2016;63:764-75.

146 Miele L, Valenza V, La Torre G, et al. Increased intestinal permeability and tight junction alterations in nonalcoholic fatty liver disease. Hepatology 2009;49:1877-87.

147 Mutlu EA, Gillevet PM, Rangwala $\mathrm{H}$, et al. Colonic microbiome is altered in alcoholism. Am J Physiol Gastrointest Liver Physiol 2012;302:G966-78.

148 Tilg $H$, Mathurin P. Altered intestinal microbiota as a major driving force in alcoholic steatohepatitis. Gut 2016;65:728-9.

149 Chen Y, Yang F, Lu H, et al. Characterization of fecal microbial communities in patients with liver cirrhosis. Hepatology 2011;54:562-72.

150 Qin N, Yang F, Li A, et al. Alterations of the human gut microbiome in liver cirrhosis. Nature 2014;513:59-64.

151 Shawcross DL, Shabbir SS, Taylor NJ, et al. Ammonia and the neutrophil in the pathogenesis of hepatic encephalopathy in cirrhosis. Hepatology 2010;51: 1062-9.

152 Bajaj JS, Ridlon JM, Hylemon PB, et al. Linkage of gut microbiome with cognition in hepatic encephalopathy. Am J Physiol Gastrointest Liver Physiol 2012;302: G168-75.

153 Dawson AM, McLaren J, Sherlock S. Neomycin in the treatment of hepatic coma. Lancet 1957;273:1262-8. 
154 Morgan MH, Read AE, Speller DC. Treatment of hepatic encephalopathy with metronidazole. Gut 1982;23:1-7.

155 Sharma BC, Sharma P, Lunia MK, et al. A randomised, double-blind, controlled trial comparing rifaximin plus lactulose with lactulose alone in treatment of overt hepatic encephalopathy. Am J Gastroenterol 2013;108: 1458-63.

156 Bass NM, Mullen KD, Sanyal A, et al. Rifaximin treatment in hepatic encephalopathy. N Engl J Med 2010:362:1071-81.

157 Mullen KD, Sanyal AJ, Bass NM, et al. Rifaximin is safe and well tolerated for long-term maintenance of remission from overt hepatic encephalopathy. Clin Gastroenterol Hepatol 2014;12:1390-7.e2.
158 Bajaj JS, Heuman DM, Wade JB, et al. Rifaximin improves driving simulator performance in a randomized trial of patients with minimal hepatic encephalopathy. Gastroenterology 2011;140:478-487.e1.

159 Maharshi S, Sharma BC, Srivastava S, et al. Randomised controlled trial of lactulose versus rifaximin for prophylaxis of hepatic encephalopathy in patients with acute variceal bleed. Gut 2015;64:1341-2.

160 Bajaj JS, Heuman DM, Sanyal AJ, et al. Modulation of the metabiome by rifaximin in patients with cirrhosis and minimal hepatic encephalopathy. PLOS ONE 2013;8: e60042.

161 http://www.fda.gov/NewsEvents/Newsroom/PressAnnouncements/ ucm206104.htm 